\title{
Influence du bec de volute sur le bruit généré par une pompe centrifuge
}

\author{
The influence of the volute tongue on the noise generated \\ by a centrifugal pump
}

par D. Descamps, G. Caignaert

Laboratoire de Mécanique de Lille (URA CNRS 1441)

The paper presents and discusses experimental results regarding the effects of some volute tongue variations (more precisely the gap between the volute tongue and the impeller outlet) for a centrifugal pump, on the pressure fluctuations at the blade passing frequency. RMS values of pressure fluctuations at blade passing frequency measured in the discharge pipe of the tested pumps are also compared to the results of a computer code based on the theoretical model proposed by Simpson, Clarck and Weir.

\section{INTRODUCTION}

Des considérations de rendement et de cavitation sont le plus souvent à la base de la définition des paramètres de conception de la roue et des parties statoriques (en particulier le diffuseur et/ou la volute) d'une pompe centrifuge. La recherche d'un bruit hydraulique aussi faible que possible, en particulier aux fréquences discrètes que sont la fréquence de passage d'aubages et ses harmoniques, constitue de plus en plus une contrainte complémentaire à prendre en compte par le concepteur de la machine. Il est pour cela nécessaire de disposer de corrélations permettant de définir, aussi rapidement et efficacement que possible, les principaux paramètres de conception, car les méthodes inverses de dessin n'intègrent pas encore les critères de bruit de façon fiable. Les bureaux d'études utilisent donc encore des données issues de statistiques bâties sur des résultats expérimentaux disponibles ([1], [2]).

Le travail analytique de Simpson, Clarck et Weir ([3]) demeure l'une des références utilisées pour la prédiction du bruit hydraulique des pompes centrifuges. Leur travail théorique permet le calcul d'une valeur efficace moyenne des fluctuations de pression, à chaque harmonique de la fréquence de rotation, dans la section de sortie d'une pompe centrifuge. Ce calcul analytique est effectué pour une machine bidimensionnelle dont les divers aubages (roue, diffuseur, volute) sont représentés par des arcs de spirales logarithmiques. Leur travail propose également une formule de corrélation plus globale, beaucoup plus simple à utiliser, mais dont les coefficients numériques ont été définis à partir de résultats d'essais, dans les conditions de rendement maximal, sur un nombre limité de pompes industrielles.
Cette formule-globale, transformée par Pempie et Metail [1] de façon à l'exprimer en fonction des caractéristiques du triangle des vitesses " moyen" en sortie de roue, a été utilisée comme base de dimensionnement pour des pompes destinées à un programme expérimental de recherche du groupe de travail " hydraulique et bruit " de la Société Hydrotechnique de France. Trois pompes centrifuges à simple volute ont ainsi été dessinées et réalisées en prenant les mêmes bases de dimensionnement (débit, hauteur interne, vitesse de rotation) mais en effectuant divers choix des paramètres de conception en sortie de roue (diamètre, largeur, angle d'aubage) et en définissant les volutes adaptées associées. Ces trois pompes ont déjà fait l'objet de nombreuses campagnes d'essais hydrauliques et hydroacoustiques, en particulier sur la boucle d'essais SESAME du Laboratoire de Mécanique de Lille, et les principaux résultats ont été publiés ([4], [5], [6], [7]).

La pompe SHF A0, au tracé le plus conventionnel, a fait l'objet d'une série d'essais complémentaires au cours desquels on a fait varier progressivement le jeu relatif entre la sortie de roue et le bec de volute, par rognages de ce bec de volute. Ces résultats expérimentaux sont ici présentés, et une partie d'entre eux fait l'objet d'une comparaison avec les prédictions issues de l'application du travail théorique de Simpson, Clark et Weir.

\section{II — PRINCIPALES CARACTÉRISTIQUES DE LA POMPE}

La figure 1 présente schématiquement la roue de la pompe SHF $\mathrm{A} 0$, dont les paramètres géométriques principaux sont les suivants : 


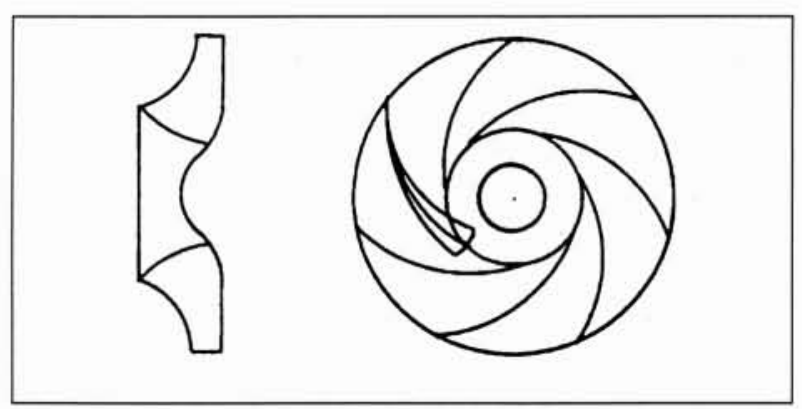

1. Schéma de la roue SHF A0.

- diamètre d'entrée en ceinture

$$
\begin{aligned}
& : D_{1}=200 \mathrm{~mm} \\
& : D_{2}=352,2 \mathrm{~mm} \\
& : b_{2}=43,6 \mathrm{~mm} \\
& : Z=7
\end{aligned}
$$$$
\text { - diamètre de sortie }
$$

- nombre d'aubes

rapport à la direction tangentielle)

$$
: \beta_{2}=22,5^{\circ}
$$

Les conditions nominales de tracé sont les suivantes :

- vitesse de rotation

$$
\begin{aligned}
& : N=1330 \mathrm{tr} / \mathrm{min} \\
& : Q_{N}=495 \mathrm{~m}^{3} / \mathrm{h} \\
& : H_{\text {in }}=32,8 \mathrm{~m}
\end{aligned}
$$$$
\text { - débit volume }
$$

- hauteur interne

Le coefficient adimensionnel de vitesse angulaire calculé au point de rendement maximal de la pompe est égal à 0,80 . Les composantes radiales et circonférentielles de la vitesse absolue moyenne de l'écoulement en sortie de roue, déduites des conditions nominales de tracé ci-dessous, ont respectivement pour valeurs $2,9 \mathrm{~m} / \mathrm{s}$ et $12,5 \mathrm{~m} / \mathrm{s}$. Il faut noter que le bord de fuite des aubages de la roue est parallèle à l'axe de rotation de la machine.

Le tracé initial de la volute est décrit très schématiquent par la figure 2 .

$$
\text { Le jeu relatif, } \frac{R_{3}-R_{2}}{R_{2}}
$$

mesuré entre la sortie de la roue et le bec de la volute est égal à $2,4 \%$. Le bord d'attaque de ce bec de volute est également parallèle à l'axe de rotation de la machine. Le tableau I et la figure 3 présentent les principales caractéristiques géométriques des modifications (B,C et D) obtenues par rognage de volute initial (A).

\begin{tabular}{|c|c|c|}
\hline Référence & $\begin{array}{c}\text { Jeu relatif } \\
\%\end{array}$ & $\begin{array}{c}\text { rayon du bec au } \\
\text { bord d'attaque }(\mathrm{mm})\end{array}$ \\
A & 2,4 & 2,4 \\
B & 3,8 & 4,0 \\
C & 5,7 & 5,7 \\
D & 7,0 & 7,8 \\
\hline
\end{tabular}

Tableau I. - Jeu relatif et rayon au bec des quatre géométries essayées.

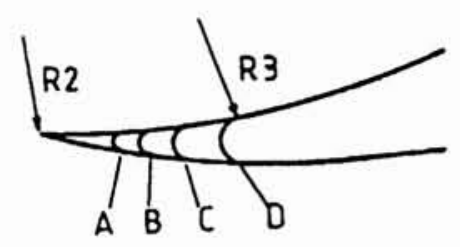

3. Schéma des quatre becs ayant fait l'objet d'essais.

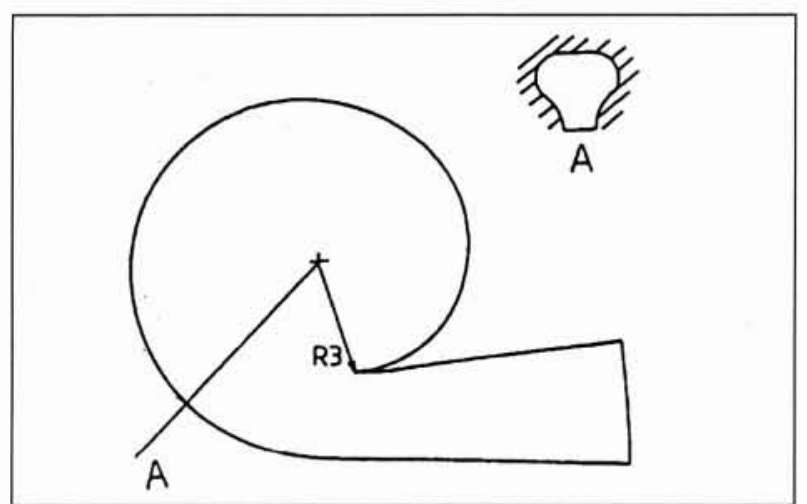

2. Volute de la pompe centrifuge SHF A0.

\section{CONDITIONS DE MESURES}

Les essais de la pompe ont été effectués sur la boucle d'essais SESAME ([4], [5]). Les conduites d'aspiration et de refoulement ont des diamètres respectivement égaux à $250 \mathrm{~mm}$ et $200 \mathrm{~mm}$. Les mesures de débit, de vitesse de rotation, du couple sur l'arbre, de la pression d'entrée et de la différence de pression entre l'entrée et la sortie permettant d'accéder aux performances hydrauliques moyennes (hauteur totale d'élévation, rendement et NPSH disponible) de chaque point de fonctionnement étudié. Les résultats présentés ici sont tous relatifs à des essais effectués hors cavitation à $1330 \mathrm{tr} / \mathrm{min}$ : la fréquence de passage d'aubages associée est donc égale à $155 \mathrm{~Hz}$.
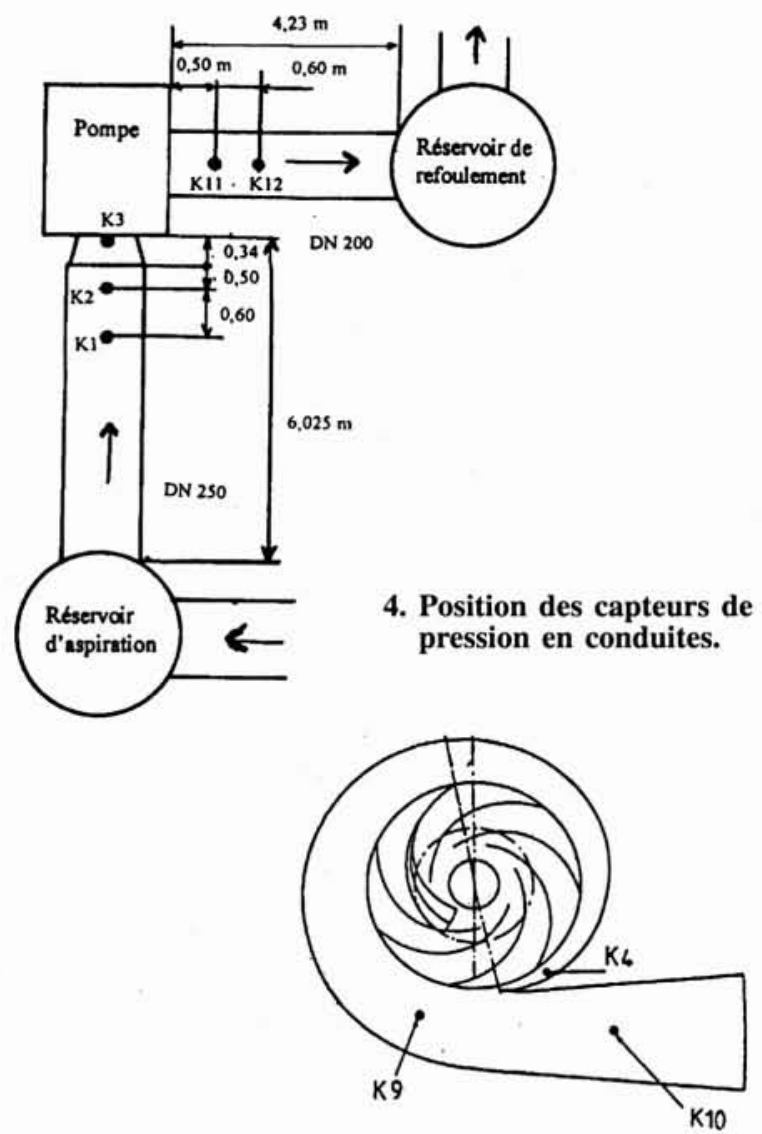

5. Position des capteurs de pression sur la volute. 


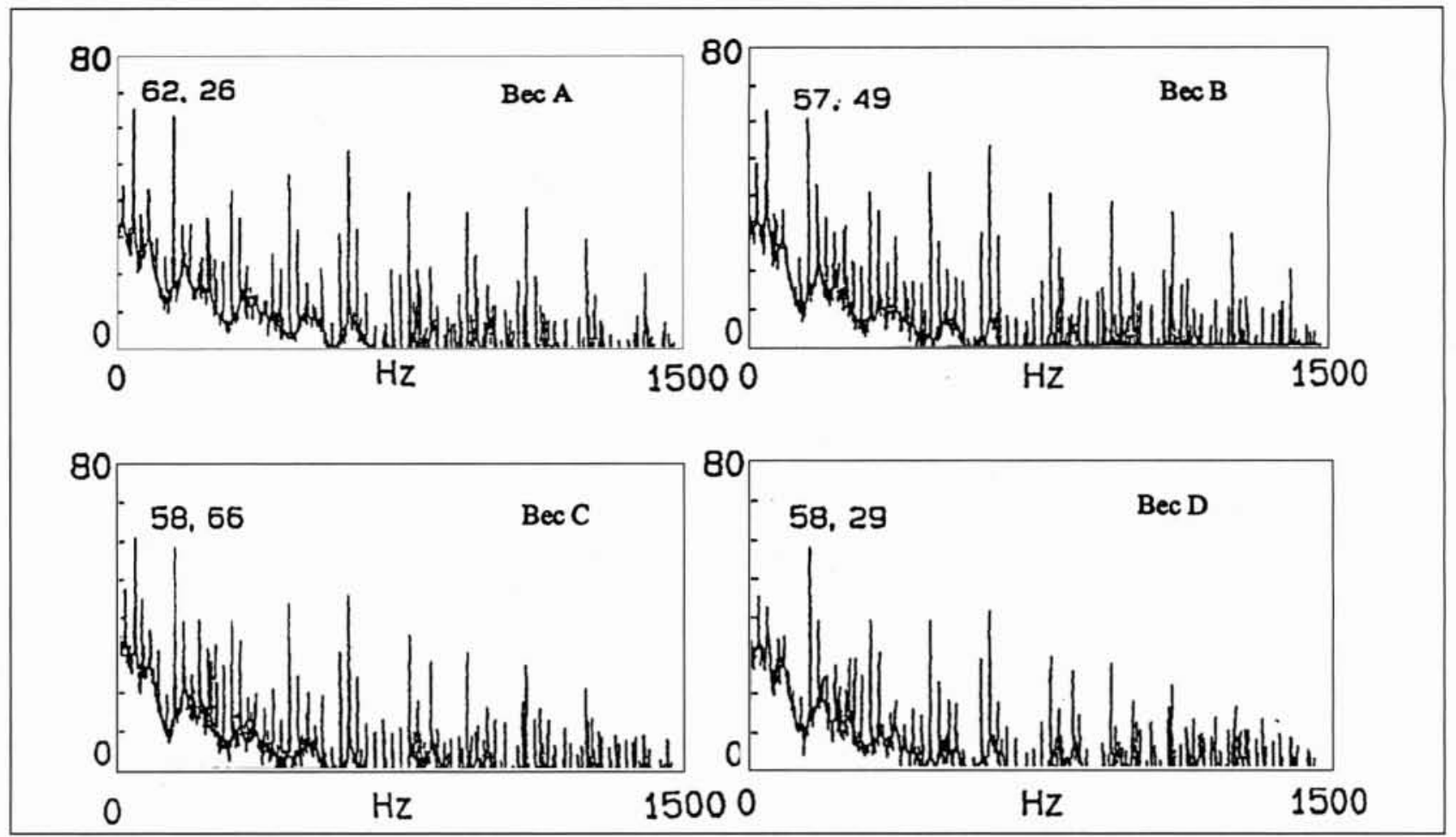

6. Autospectres des fluctuations de pression au refoulement de la pompe (capteur K12), dans les conditions nominales de fonctionnement $\left(495 \mathrm{~m}^{3} / \mathrm{h}\right.$ et $\left.1330 \mathrm{tr} / \mathrm{min}\right)$. Les valeurs efficaces en ordonnées sont exprimées en $\mathrm{dB}$ avec une référence à $1 \mathrm{~Pa}$. La valeur obtenue à la fréquence de passage d'aubages $(155 \mathrm{~Hz})$ est indiquée dans chacune des quatre figures qui correspondent aux quatre becs essayés.

Les fluctuations de pression en divers points de l'installation (voir figure 4) ont été mesurées à l'aide de capteurs piézoélectriques montés en membrane affleurante sur la conduite d'aspiration (capteurs K1 et K2), sur la conduite de refoulement (capteurs K11 et K12) ainsi qu'en divers points du carter de pompe (capteur K3 en entrée de pompe, juste en sortie du cône d'entrée ; capteurs K4, K9 et K10 sur la paroi de la volute comme indiqué sur la figure 5). Les fluctuations du couple sur l'arbre ont également été mesurées et analysées mais ces résultats ne sont pas présentés ici.

Les résultats présentés et discutés ici sont limités à l'exploitation des valeurs efficaces des fluctuations de pression à diverses fréquences discrètes, en particulier la fréquence de passage des aubages, car les traitements effectués font bien apparaître des raies très fines émergeant nettement des autospectres enregistrés comme le montre bien la figure 6. Il faut noter que les huit autospectres de fluctuations de pression ont été mesurés simultanément et sont le résultat d'une analyse dans la gamme de fréquences $0-1500 \mathrm{~Hz}$ effectuée avec une fréquence d'échantillonnage égale à $4000 \mathrm{~Hz} ; 8192$ échantillons sont utilisés pour déterminer les autospectres avec utilisation d'une fenêtre de Hanning et d'une moyenne sur 10 spectres.

Le caractère particulier des divers autospectres de fluctuations de pression, avec des raies très fines liées à la fréquence de rotation et ses harmoniques, est également très affirmé aux divers autres points de mesures comme le confirment les figures 7 (mesures en conduite d'aspiration) et 8 (mesures près du bec de volute). On peut par contre noter, en observant les figures 6,7 et 8 , les différences très importantes entre les niveaux mesurés en conduites (on a ici des résultats de mesures qui caractérisent bien les phénomènes propagatifs dans l'installation) et les niveaux mesurés dans la zone d'interaction forte entre parties fixes et mobiles (fluctuations au caractère plus local, mais qui sont mesurées au voisinage direct de la zone de la source principale de bruit hydraulique dans l'installation).

\section{IV — PERFORMANCES HYDRAULIQUES}

Des modifications du jeu entre le bord de fuite des aubages de la roue et le bec de volute ainsi que de la géométrie du bec de volute (rayon au bord d'attaque, angle du profil moyen du bec par rapport à la direction tangentielle,...) peuvent avoir une influence plus ou moins grande sur la forme de la caractéristique hauteur totale d'élévation-débit de la pompe, particulièrement dans les fonctionnements à débits partiels [8]. Les figures 9 et 10 montrent l'effet des rognages effectués sur, d'une part, la caractéristique hauteur totale d'élévation-débit et, d'autre part, la courbe rendementdébit de la pompe. Comme on peut le constater, l'augmentation du jeu entre la sortie de roue et le bec de volute se traduit par une légère augmentation des hauteurs totales d'élévation, plus particulièrement dans les fonctionnements à débits partiels et par une très légère diminution du rendement maximal de la pompe. Ces modifications du jeu n'ont pas d'influence sensible sur la valeur du débit critique de recirculation en entrée de pompe $(\mathrm{QK} / \mathrm{QN}=0,54)$, ce qui parait tout à fait normal. 


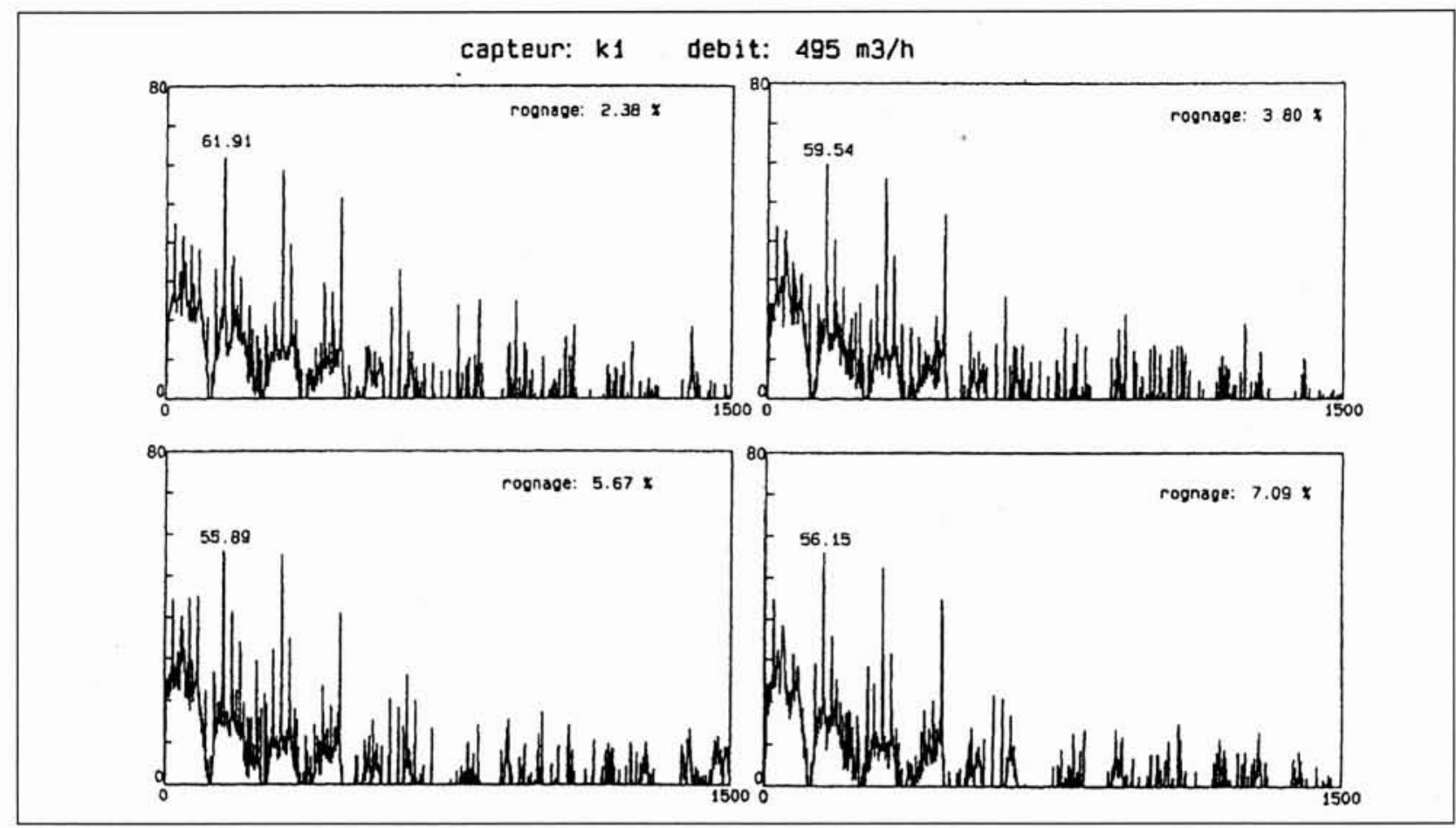

7. Autospectres des fluctuations de pression à l'aspiration de la pompe (capteur K11), dans les conditions nominales de fonctionnement $\left(495 \mathrm{~m}^{3 /} / \mathrm{h}\right.$ et $\left.1330 \mathrm{tr} / \mathrm{min}\right)$.

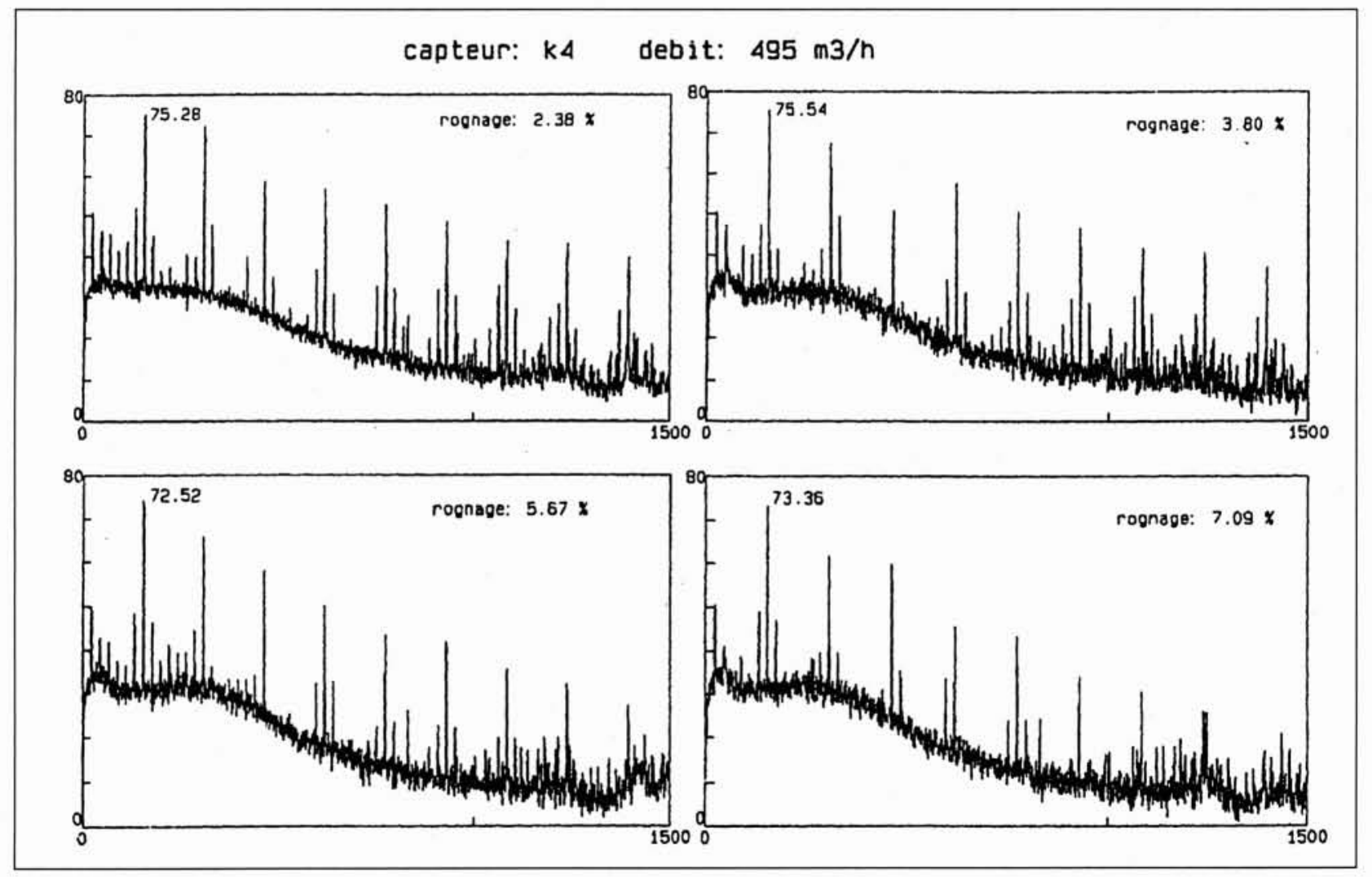

8. Autospectres de fluctuations de pression près du bec de volute (capteur K4), dans les conditions nominales de fonctionnement $\left(495 \mathrm{~m}^{3} / \mathrm{h}\right.$ et $\left.1330 \mathrm{tr} / \mathrm{min}\right)$. 
hauteur totale d'élévation (m)

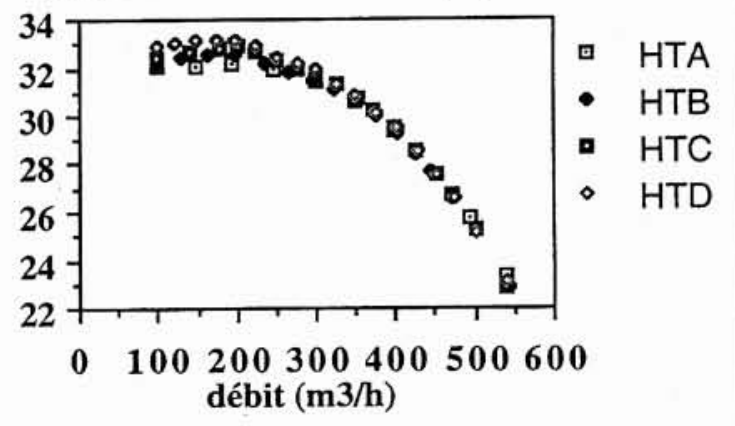

9. Courbes hauteur totale d'élévation-débit pour les 4 géométries de bec de volute essayées

\section{V — FLUCTUATIONS DE PRESSION À LA FRÉQUENCE DE PASSAGE D'AUBAGES}

La figure 11 permet d'apprécier l'influence des rognages du bec de volute sur les fluctuations de pression, à la fréquence de passage des aubes, en divers points de la volute (K4, K9 et K10) et un point de la conduite de refoulement (K12). Au niveau du capteur K4, proche du bec de volute, on n'observe pas d'évolution très significative avec les 4 becs testés ; dans cette portion de la volute, les fluctuations de pression sont directement associées aux interactions entre l'écoulement non uniforme sortant de chaque canal interaubes et le bec de volute comme cela a par exemple été mis en évidence dans la référence [9] à l'aide d'essais sur cette même roue SHF A0 et de la volute dans sa configuration initiale (A) : l'intensité de ces interactions évolue avec le

\section{rendement}

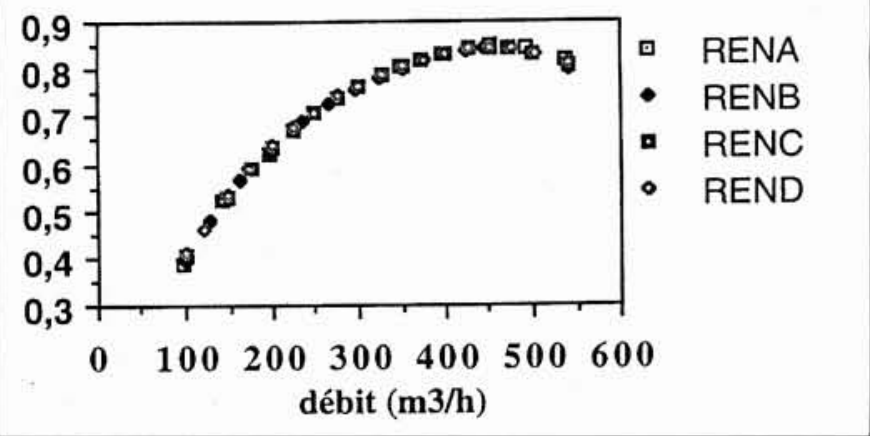

10. Courbes rendement-débit pour les 4 géométries de bec de volute essayées

point de fonctionnement de la pompe, du fait, en particulier, des évolutions de l'incidence de l'écoulement par rapport au profil que constitue le bec de volute, et passe par un minimum pour un débit relatif $\mathrm{Q} / \mathrm{QN}$ égal à 0,82 , ce qui est inférieur au débit relatif de meilleur rendement dont la valeur est égale à 0,91 . On peut peut-être relier cet écart au fait que le débit optimal correspondant au minimum de pertes hydrauliques, est légèrement différent pour la roue et la volute [10]. Près de la sortie de la volute (capteur K9) et sur le cône de sortie (capteur K10), l'influence du bec de volute devient plus significative et se traduit par une diminution assez générale du niveau de fluctuation de pression à mesure que le jeu relatif augmente, avec un écart de l'ordre de $6 \mathrm{~dB}$ au débit nominal QN et aux débits partiels, et avec un écart un peu plus faible au point de meilleur rendement. Sur ces deux capteurs, $\mathrm{K} 9$ et $\mathrm{K} 10$, on peut par ailleurs observer que le
dB (ref. 1 Pa)

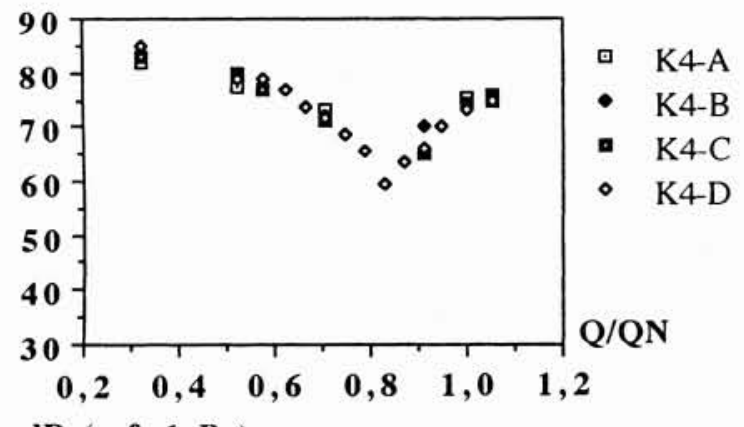

dB (ref. 1 Pa)

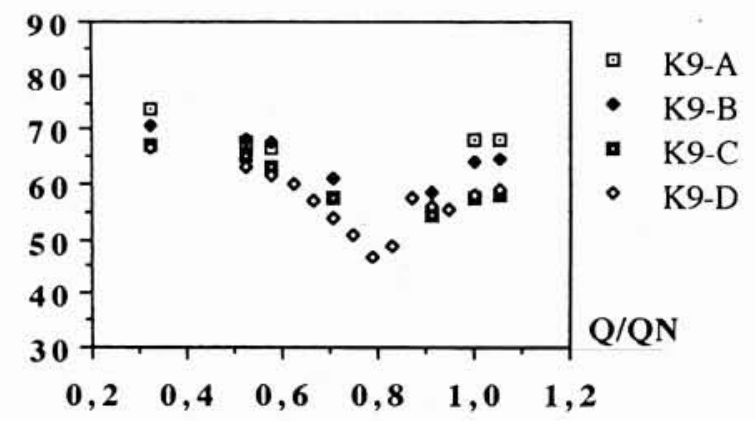

dB (ref. 1 Pa)

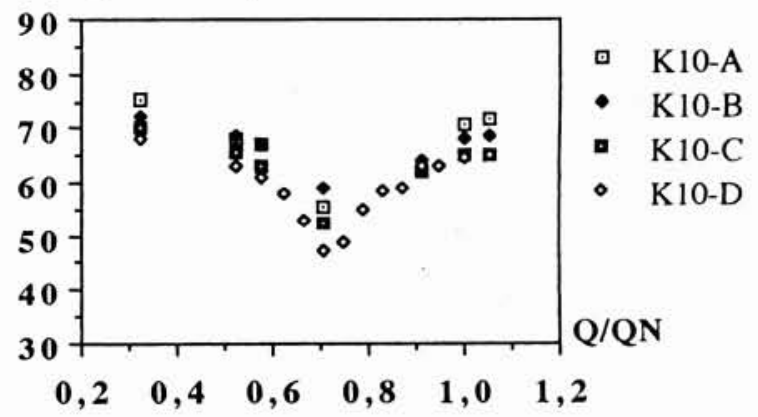

dB (ref. 1 Pa)

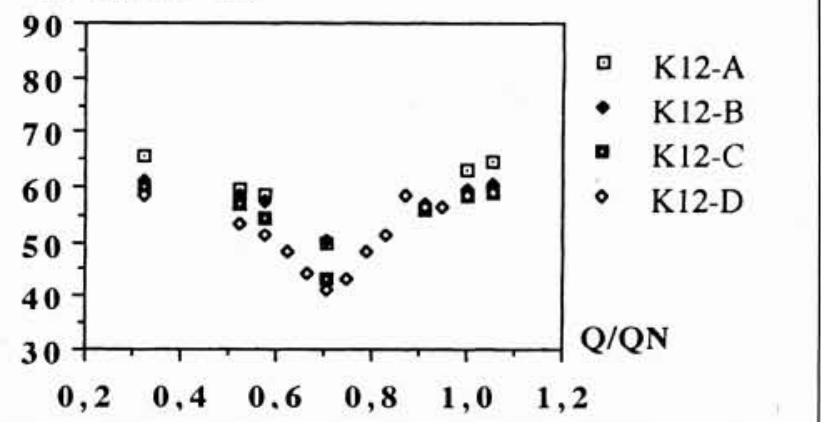

11. Valeurs efficaces des fluctuations de pression, à la fréquence de passage d'aubages, mesurées sur les capteurs $\mathrm{K} 4$, K9, K10 et K12 (essais à $1330 \mathrm{tr} / \mathrm{min}$ ). 


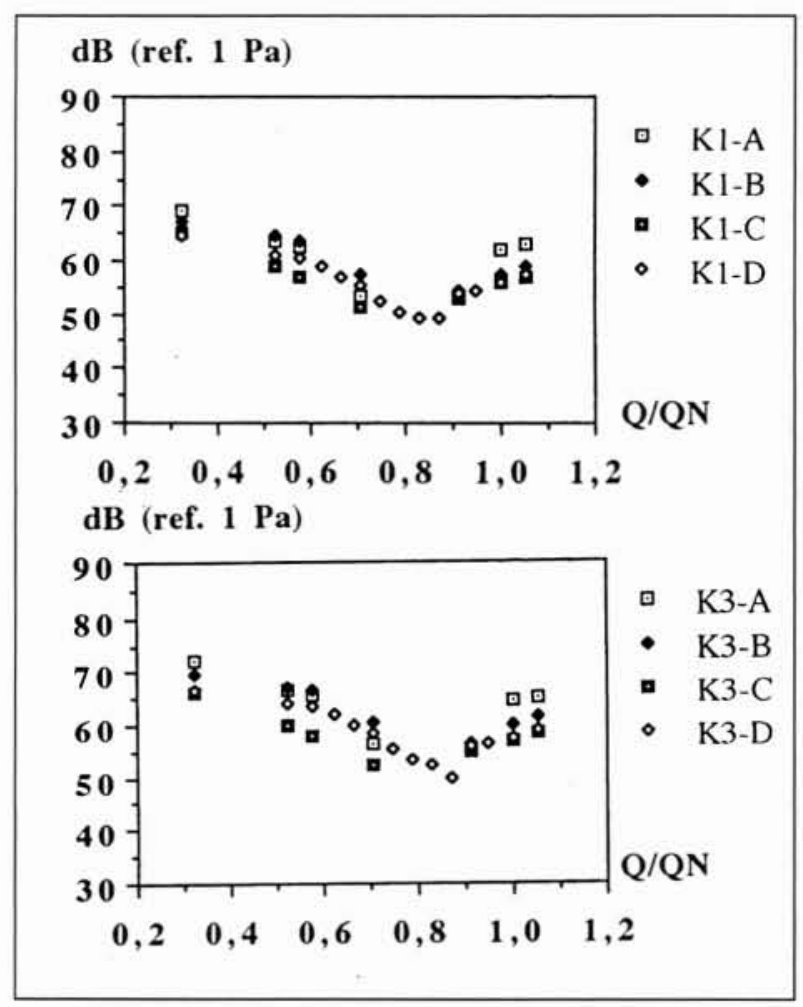

12. Valeurs efficaces des fluctuations de pression, à la fréquence de passage d'aubages, mesurées sur les capteurs K3 et K1 (essais à $1330 \mathrm{tr} / \mathrm{min}$ ).

niveau minimal de fluctuations de pression est obtenu pour des valeurs encore plus faibles du débit relatif $(\mathrm{Q} / \mathrm{QN}=0,79$ pour $\mathrm{K} 9$ et $\mathrm{Q} / \mathrm{QN}=0,71$ pour $\mathrm{K} 10$ ), ce qui peut être associé au fait qu'il y a dans cette zone une organisation progressive du régime d'ondes qui s'installe dans l'ensemble du circuit.

Dans la conduite de refoulement (capteur K12), les évolutions sont très voisines de celles observées pour le capteur $\mathrm{K} 10$; les écarts sur les niveaux mesurés sur les capteurs $\mathrm{K} 10$, K11 et K12 peuvent assez facilement être associés aux caractéristiques du régime d'ondes obtenu dans la tuyauterie de refoulement qui, il faut le noter, n'est pas munie d'une T.A.L. en son extrémité mais pour laquelle on peut estimer la valeur du coefficient de réflexion des ondes en extrémité à environ 0,70 pour la fréquence étudiée [5].

La figure 12 résume les résultats obtenus à l'entrée de la pompe, juste en face de la roue en sortie du cône d'entrée (capteur K3) et en paroi de la conduite d'aspiration (capteur $\mathrm{K} 1$ ). Le niveau minimal de fluctuations de pression à la fréquence de passage d'aubages est cette fois obtenu au voisinage direct du point de meilleur rendement de la machine $(\mathrm{Q} / \mathrm{QN}=0,91)$. Au dessus du débit nominal, le niveau décroît lorsque le jeu entre la sortie de roue et le bec de volute augmente : les variations observées sont toutefois beaucoup moins importantes que celles qui sont mesurées au refoulement de la pompe. Dans les fonctionnements à débits partiels, les évolutions sont plus difficiles à interpréter et les effets de l'apparition des recirculations devront être analysés plus en détail. Il faut également rappeler ici que les mesures à l'aspiration s'effectuent dans des conditions très réverbérantes au niveau du réservoir d'aspiration.

\section{VI — COMPARAISON AU MODÈLE DE SIMP- SON, CLARK ET WEIR ([3])}

Un code de calculs, fondé sur le modèle théorique complet de Simpson, Clark et Weir [3], a été développé au laboratoire par M. Jaremczak [10]. Le modèle s'applique à une pompe bidimensionnelle dont les aubages de roue, de diffuseur et la volute sont représentés par des arcs de spirales logarithmiques. Les valeurs RMS des fluctuations de pression en sortie de pompe peuvent être calculées à n'importe quel multiple de la fréquence de rotation. Ces fluctuations de pression sont liées à deux effets :

- d'une part les interactions entre grilles d'aubes fixes et grilles d'aubes mobiles, résultats d'un calcul analytique dans lequel les effets visqueux sont négligés,

- d'autre part les interactions entre le sillages des aubages mobiles (roue de la pompe) et les aubages fixes (diffuseur, volute) ; à ce niveau le modèle de sillage proposé par Kemp et Sears [11] est utilisé.

L'application à une pompe réelle s'effectue par définition d'une pompe bidimensionnelle " équivalente" :

- la largeur en sortie de roue, b2, est choisie comme épaisseur constante de la pompe "équivalente",

- l'angle d'aubage réel en sortie de roue, $\beta 2$, est choisi comme angle des arcs de spirales logarithmiques qui constituent les aubages de la roue de la pompe " équivalente "; ces arcs de spirales logarithmiques s'étendent du rayon de sortie réel de la pompe étudiée, R2, à un rayon d'entrée moyen de la pompe,

- une volute bidimensionnelle, " équivalente " à la volute réelle, est définie par une spirale logarithmique s'étendant du rayon réel au bec à un rayon de sortie calculé par référence à une distribution de vitesses de type vortex libre dans la section de sortie de la volute,

- le coefficient de traînée d'un aubage de roue, nécessaire pour la prise en compte des effets visqueux dans le modèle, est estimé à partir de la valeur des pertes dans la roue ; ces pertes dans la roue, qui évoluent avec le débit, ont été déterminées expérimentalement à partir des mesures de couple et de mesures des distributions de pressions moyennes en entrée de volute ou sortie de roue.

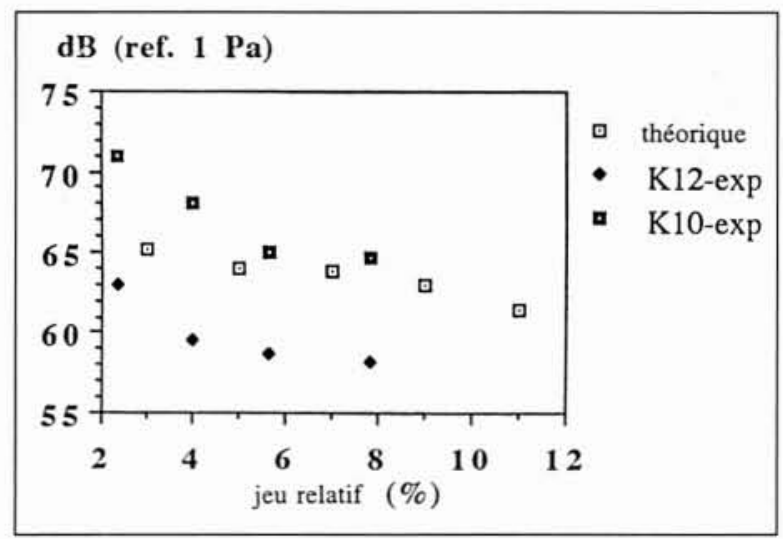

13. Comparaison des valeurs efficaces, des fluctuations de pression, à la fréquence de passage des aubes, calculées et mesurées au refoulement de la pompe, dans les conditions nominales de fonctionnement $\left(495 \mathrm{~m}^{3} / \mathrm{h}\right.$ et $\left.1330 \mathrm{tr} / \mathrm{min}\right)$. 
Le code de calculs ainsi élaboré a été utilisé par $\mathrm{M}$. Jaremczak [3] pour analyser l'influence d'une augmentation du jeu entre sortie de roue et bec de volute de la pompe SHF $\mathrm{A} 0$, dans les conditions nominales de fonctionnement $(\mathrm{Q}=$ $495 \mathrm{~m}^{3} / \mathrm{h}$ et $\left.\mathrm{N}=1330 \mathrm{tr} / \mathrm{min}\right)$. Les résultats obtenus, à la fréquence de passage des aubes, sont présentés sur la figure 13 où on a porté également les résultats expérimentaux, pour les quatre becs (A,B,C,D), aux points de mesure K10 (cône de sortie de la pompe) et K12 (conduite de refoulement). Comme on peut le constater, l'allure globale de l'évolution mesurée est bien représentée par le calcul issu du modèle de Simpson, Clark et Weir. Les écarts entre les capteurs K10 et K12 sont dus, d'une part, à l'effet du diffuseur conique situé entre la section de sortie de la volute et l'entrée de la conduite de refoulement et, d'autre part, au fait que les conditions de mesures au refoulement ne sont pas anéchoïques, ce qui entraîne automatiquement des écarts de niveaux de fluctuations de pression tout au long de cette conduite.

\section{VII $\square$ CONCLUSION}

Les résultats présentés ici montrent bien l'effet positif d'une augmentation du jeu entre la sortie de la roue et le bec de la volute sur la diminution du niveau des fluctuations de pression en sortie de pompe. Ces résultats confirment ceux obtenus par d'autres auteurs [3], et montrent bien la capacité d'un modèle analytique simplifié tel que celui proposé par Simpson, Clark et Weir [3] à rendre compte qualitativement de cet effet aux fréquences discrètes de calcul, harmoniques de la fréquence de rotation et donc d'être utilisable lors des étapes de choix des principaux paramètres de conception de ce type de machine en vue de répondre à des contraintes de réduction du bruit hydraulique.

Une validation complémentaire sera effectuée prochainement par l'intermédiaire d'essais sur les deux autres pompes du programme expérimental de la SHF (pompes SHF A2 et C0). Par ailleurs, ces travaux seront complétés par la mise en œuvre d'une approche hydroacoustique plus globale permettant de caractériser les évolutions de la matrice de transfert de la pompe et des termes sources [12] lorsqu'on procède à des variations paramétriques au niveau du bec de volute. Un couplage avec l'utilisation de mesures de fluctuations de pressions au sein d'un canal inter-aubes de la roue sera utilisé pour aider à l'analyse des résultats.

\section{RÉFÉRENCES BIBLIOGRAPHIQUES}

[1] Pempie, P. and Métail, J.P. - Prédiction du bruit des pompes centrifuges. La Houille Blanche, 1985, 5, p. 635-654.

[2] Deeprose, W.H. and Bolton, A.N. - Current industrial pumps and fan fluid-borne noise level prediction. IMechE publication " Limiting noise from pumps, fans and compressors ", 1977-10, paper C251/77, p 43-50.

[3] Simpson, H.C., Clark, T.A. and Weir, G.A. - A theoretical investigation of hydraulic noise in pumps. Journal of Sound and Vibrations, 1967, 5 (3), p. 456-488.

[4] Caignaert, G., Kermarec, J., Trollat, C., and Jaremczak, M. - Hydraulic noise of centrifugal pumps : comparison of three different pumps designed for the same duty point. Proceedings of the 15th Symposium of the IAHR ; Beograd ; Septembre 1990 ; Paper T4 (12 pages).

[5] Caignaert, G., Desmet, B., Barrand, J.P., Ouaked, R., and Stevenaert, D. - Experimental determination of hydraulic noise of centrifugal pumps. Internoise $88 ; 30$ août - 1er septembre 1988; Avignon : France ; p. 667-670.

[6] Caignaert, G., Ghelici, N., Stevenaert, D., and Barrand, J.P. - Utilisation de l'intensimétrie en conduite pour la caractérisation du bruit hydraulique généré par une pompe centrifuge dans un circuit. Journal de Physique ; Vol 51 ; 1990 ; p. C2 1185-C2 1188.

[7] Jaremczak, M. and Caignaert G. - Theoretical and experimental analysis of hydraulic noise of three centrifugal pumps. 1st international symposium " Pump Noise and Vibrations " : Clamart ; $7-9$ july 1993 ; p. 103-110.

[8] Caignaert, G. - Influence de la géométrie de l'environnement aval de la roue sur les caractéristiques des pompes à débit partiel. La Houille Blanche ; $n^{\circ} 2 / 3 ; 1982 ;$ p. $131-138$.

[9] Chatel, G., Jolly, C., Lauro, J.F. - Analysis of Noise Generation Mechanism in a Centrifugal Pump. ASME 1995 Fluid Machinery Meeting. South Carolina (USA), FED-Vol 222.

[10] Jaremczak, M. - Analyse théorique et expérimentale du bruit hydraulique de pompes centrifuges. Thèse soutenue à l'Université des Sciences et Technologies de Lille, 15 Juillet 1992.

[11] Kemp, N.H, Sears, W.R. Aerodynamics Interference Between Moving Blade Rows. Journal of Aeronautical Sciences, Vol. 20, n 9, 1953, p. 585-597.

[12] Stirneman, A., Eber, J., Bolleter, U. and Pace, S. - Experimental determination of the dynamic transfer matrix for a pump. Trans. ASME, J. of Fluids Engineering : vol 109, $\mathrm{n}^{\circ} 3$, sept 1987, p. 218-225. 